COMMENTARY ON COVID-19 AND THE FoOd SYSTEM

\title{
Communication helped UConn Extension address the needs of agricultural producers
}

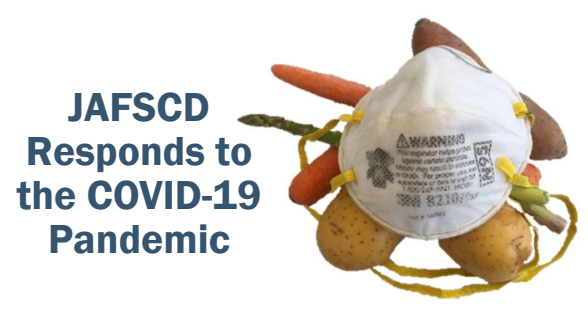

\author{
Stacey F. Stearns a $*$ \\ University of Connecticut Extension \\ Joseph A. Bonelli b \\ University of Connecticut Extension
}

Submitted September 17, 2020 / Published online October 19, 2020

Citation: Stearns, S. F., \& Bonelli, J. A. (2020). Communication helped UConn Extension

address the needs of agricultural producers. Journal of Agriculture, Food Systems, and Community

Development, 10(1), 201-203. https://doi.org/10.5304/jafscd.2020.101.008

Copyright $(C) 2020$ by the Authors. Published by the Lyson Center for Civic Agriculture and Food Systems. Open access under CC-BY license.

$\mathrm{T}$ he worldwide pandemic due to COVID-19 (coronavirus) has produced unprecedented challenges around the world. Agricultural producers were still working on farms, in greenhouses, and along the coast in Long Island Sound during the early stages of the COVID-19 outbreak. Severe challenges related to labor, food safety, on-farm biosecurity, marketing, and distribution confronted producers. University of Connecticut (UConn) Extension educators understood that our audiences' needs had changed drastically, and communication would help us understand those changes and provide the services required for the success of their operations.

We addressed these challenges using a multifaceted approach. Our team curated resources on a single website for our statewide audiences, including agricultural producers. Extension educators developed resources for specific agricultural sectors, such as fruit and vegetable farms, aquaculture, and nursery and landscape professionals. Links to important updates from the Connecticut Department of Agriculture were also added to our website.

We added new information to the website as it became available. Agricultural producers received regular updates from Extension through multiple channels, including email, social media, other websites, and word of mouth. Resources on the website for all types of audiences include information on food safety and cooking, hand-washing and sanitizers, infection protection, financial advice, and listings of

\footnotetext{
a * Stacey F. Stearns, Program Specialist-Educational Outreach, University of Connecticut Extension; 1376 Storrs Road, Unit 4134; Storrs, CT 06269-4134 USA; +1-860-486-9228; Stacey.Stearns@uconn.edu

b Joseph A. Bonelli, Associate Extension Educator, University of Connecticut Extension; Joseph.Bonelli@uconn.edu
} 
farms that are open to the public, farmers markets, and school emergency meal distribution options for children.

UConn Extension conducted surveys to assess the impact of the COVID-19 pandemic on Connecticut agriculture. The survey for agricultural producers was based on a survey developed by our Connecticut Sea Grant program for aquaculture producers. Other members of the UConn Extension team developed and implemented a similar survey for school grounds managers.

The survey for agricultural producers was anonymous and sent to farmers and growers in Connecticut. We had 178 responses to the March survey and 103 respondents to the follow-up survey in June. The institutional review board at the University of Connecticut determined that approval for the survey was not needed.

The results of the second survey showed that $47 \%$ of the respondents grew two or more commodities in their operation. Economic viability remained a concern for all businesses during the pandemic, including agricultural operations. Of the respondents, $47 \%$ indicated that farm income was up, while $28 \%$ indicated that income was down due to the pandemic. There was a wide range in the percent increase and decrease in farm income reported. The average income increase was 54\%, and the average decrease was $61 \%$.

We asked respondents about changes made to their agricultural operation in response to COVID-19. These included closing the farm to the public; limiting the number of volunteers; establishing contactless pickup and online pre-ordering; purchasing additional freezers to meet demand; requiring social distancing, use of hand sanitizers and masks; adding a new entrance; and investing in packaging and equipment for online sales.

Employee numbers closely aligned with responses to income. Eighteen percent of the respondents added between one and three employees, while $17 \%$ decreased the number of employees by two to five full- or part-time employees. Labor challenges cited by respondents included struggling to find extra help due to higher demand, health concerns, and the willingness of employees to practice sanitary practices.

Transportation has been an area of greater challenge for agricultural operations during COVID-19. Many of our respondents (56\%) said they experienced difficulties securing supplies because of the pandemic. Challenges transporting products off their agricultural operation negatively affected $75 \%$ of respondents.

Negative effects on farm markets were minimal. Only 17\% of respondents lost marketable product. We asked if customers were finding it difficult to pay invoices, and $72 \%$ of respondents said that none of their customers had had difficulties making payments.

Marketing products remains key to economic viability, and $65 \%$ of respondents had considered alternative marketing strategies as of June 2020. These include using an open food network for connecting consumers and farmers, offering online ordering and/or delivery options, increasing wholesale sales, adding food trucks, implementing pre-ordering, using social media, and participating in the USDA Food Box program.

We asked about the communication that farmers receive from Extension and our partners. The majority (84\%) of agricultural operators who responded to our survey were receiving information in a timely manner. Respondents did request improvements in communication. They wanted to increase the availability of informational webinars. They suggested that farmers market status updates were useful. Respondents also requested a reduction in the number of emails with duplicate information.

Agricultural producers cited many concerns regarding COVID-19. Extension educators and our partners can address some of these concerns. These include financial risk management, understanding customers, establishing safety protocols and signage, and understanding regulations and recommendations. 
Extension is modifying our programs to meet agricultural producers' needs. Our team refined our educational outreach resources and developed new materials to meet the challenges agricultural operators are facing. We streamlined communications among members of our agricultural Extension team since many respondents have multiple commodities. This helped reduce the number of duplicate emails that producers receive. Extension led an effort with the Connecticut Farm Bureau and Connecticut Department of Agriculture to create farm signs addressing biosecurity. Producers can purchase these signs at our cost, with no mark-up.

Extension educators continue curating information for producers and facilitating educational outreach to ensure that Connecticut's agricultural producers have all the information they need to operate their business successfully and remain economically viable. Our educators are using digital tools to connect with producers for farm visits, webinars, online classes, one-on-one consultations, crop scouting, and other services.

Agriculture is a challenging industry, yet farmers in Connecticut have remained resilient for centuries. Our role as Extension educators is to provide the resources and knowledge that will enhance their resilience and lead to their businesses' success. The COVID-19 pandemic is unprecedented, yet we can communicate with our agricultural producers and pivot the programs and resources available to help them address the challenges they are facing.

Agricultural producers are still adjusting to the challenges caused by the pandemic. UConn Extension educators are continuing to communicate with our audiences, adjust our programs to meet their needs, and support a strong and resilient agricultural community. 